\title{
High resolution computed tomographic evaluation of bronchial wall thickness in healthy and clinically asthmatic cats
}

\author{
Sungjun WON ${ }^{1,2)}$, Sookyung YUN ${ }^{2)}$, Jeosoon LEE'), Mikyung LEE'1), \\ Mincheol $\mathrm{CHO}^{2)}$ and Junghee $\mathrm{YOON}^{2) *}$ \\ 1)Irion Animal Hospital, Seoul 06015, Korea \\ ${ }^{2)}$ College of Veterinary Medicine and the Research Institute for Veterinary Science, Seoul National University, \\ Seoul 08826, Korea
}

\section{J. Vet. Med. Sci.}

79(3): 567-571, 2017

doi: 10.1292/jvms.16-0476

Received: 11 September 2016 Accepted: 17 January 2017 Published online in J-STAGE: 5 February 2017
ABSTRACT. The objective of study is to determine the thickness of bronchial walls of clinically diagnosed asthmatic cats using high resolution computed tomography (HRCT) compared to that of healthy cats. The bronchial walls and pulmonary arteries were measured in healthy 16 cats and clinically asthmatic 4 cats. The bronchial walls and pulmonary arteries were measured under general anesthesia with positive pressure inspiration using HRCT. In healthy and asthmatic cats, bronchial lumen to the artery ratio (BA ratio), the ratio of bronchial wall thickness to bronchial diameter (TD ratio) and ratio of bronchial wall thickness to pulmonary artery (TA ratio) were measured. The mean BA ratio, TD ratio and TA ratio in healthy cats were $0.86 \pm 0.12,0.18 \pm 0.02$ and $0.25 \pm 0.05$, respectively. Under the same condition, the mean BA ratio, TD ratio and TA ratio in asthmatic cats were $0.93 \pm 0.21,0.22 \pm 0.24$ and $0.37 \pm 0.06$. The TD ratio and TA ratio in asthmatic cats were significantly higher than healthy cats $(P<0.001)$. BA ratio was not significantly different in both groups $(P>0.05)$. The evaluation of bronchial wall thickness by HRCT could be useful for diagnosis of disease of bronchial wall thickening, such as feline asthma.

KEY WORDS: bronchial wall thickness, cat, feline asthma, high resolution computed tomography

Asthma is a common inflammatory disease of the airway characterized by variable and recurring symptoms, reversible airflow obstruction and bronchospasm in human medicine [2]. It has been reported that chronic inflammations of bronchial airway can cause changes to bronchial wall thickening histologically [7]. Acute symptoms of asthma usually arise from bronchospasm responsive to bronchodilator therapy. Acute and chronic inflammation not only can affect airway caliber and airflow, but also underlie excessive bronchial responsiveness and enhance the susceptibility to bronchospasm [2].

In veterinary medicine, feline asthma has been used to define a respiratory condition in cats characterized by the combination of recurrent episode of paroxysmal coughing, wheezing and dyspnea that respond to glucocorticoid therapy [4]. Asthma has been experimentally induced in cats as an animal model for human asthma. In a study of experimentally induced asthma in cats as an animal model, feline asthma is considered to be similar to allergic asthma in human in laboratory and histopathologic aspects [12]. Definite diagnosis of asthma in human can be supported by simple pulmonary function test to measure how much air and how quickly patients can blow out of their lungs with spirometry. Diagnosis of asthma in human can also be supported by methacholine challenge test and allergy test, such as inhalation allergen test and intradermal skin test $[3,8,15]$. Using bronchial provocation, such as methacholine challenge test, is limited in cats, because it depends on the compliance and cooperation of patients. It is not readily applicable to cats [4]. Therefore, the diagnosis of feline asthma is typically based on changes on plain radiography and therapeutic response to glucocorticoid after excluding other disease, such as pneumonia, neoplasia, infectious bronchitis and cardiac disease $[1,13]$.

In human medicine, computed tomography (CT) is a useful modality to assess the severity of asthma and diagnose the complications of asthma. To delineate bronchial image in CT, high resolution computed tomography (HRCT) has been used in human medicine [14]. HRCT is a scan protocol that is maximizing spatial resolution result to minimize the partial volume effect and use bone reconstruction algorithm in place of lung algorithm to maximized sharpness. Optimizing spatial resolution by employing the narrowest feasible collimation beam and a high spatial frequency reconstruction algorithm has improved resolution such that features of 100-200 $\mu \mathrm{m}$ size can be identified, allowing assessment of small airways in the region of 1.5-2 mm diameter [19]. Measurement for internal size of bronchi using HRCT has been found to be highly reproducible [17]. When HRCT was used

*Correspondence to: Yoon, J., College of Veterinary Medicine and the Research Institute for Veterinary Science, Seoul National University, 1 Gwanakro, Gwanak-gu, Seoul 08826, South Korea. e-mail: heeyoon@snu.ac.kr

(C2017 The Japanese Society of Veterinary Science

This is an open-access article distributed under the terms of the Creative Commons Attribution Non-Commercial No Derivatives (by-nc-nd) License. (CC-BY-NC-ND 4.0: https://creativecommons.org/licenses/by-nc-nd/4.0/) 
to assess airway wall thickness in chronic asthma, reproducibility was found to be high [9]. In addition, correlation between the severity of asthma and CT findings, such as bronchiectasia and emphysema, has been reported [14].

In veterinary medicine, HRCT has been used to assess bronchial wall and thoracic structures. HRCT has been used to determine the bronchial lumen to artery ratio (BA ratio) in cats [16]. Conventional CT has been used to determine the bronchial wall to adjacent arterial ratio in dogs with bronchiectasis in a retrospective manner [18]. However, the normal bronchial wall thickness or the indices of bronchial wall in cats have not yet been established.

Therefore, the objectives of this study were to use HRCT to determine the bronchial wall thickness in healthy cats and to identify bronchial wall changes between healthy cats and clinically diagnosed asthmatic cats.

\section{MATERIALS AND METHODS}

Sixteen client-owned cats, presented to the Irion Animal Hospital, Seoul, Korea, over a period of 7 months (November 2014 -May 2015) were included in the study. The study population consisted of client owned clinically healthy cats. All of these cats were undergoing minor dental treatment, and all of owners of these cats were voluntarily participated in this study. The mean age of cats was 3.8 years (range 1-7 years) with a mean body weight of $4.0 \mathrm{~kg}$ (range $2.8-6.0 \mathrm{~kg}$ ). Sixteen cats were Domestic short hair, and 1 cat was Persian cat. All cats were considered clinically healthy except for dental disease, by the owners at the time of the investigation, and this observation was supported by findings on physical examination, complete blood count, serum chemistry and thoracic radiography. After CT examination, microscopic examination via tracheal washing was performed, and animal with suspected infectious condition was excluded.

Four asthmatic cats were selected from patients who have visited Irion animal hospital from 2014 to 2015 , and they showed clinical signs of wheezing and coughing, obvious bronchial and interstitial pattern in radiograph and therapeutic response to glucocorticoid. The mean age of cats was 7.8 years old (range 5-11 years old) with a mean body weight of 5.2 kg (range 2.7-7.0 $\mathrm{kg}$ ). Four cats were 2 Russian Blue cats, 1 Abyssinian cat and 1 Domestic short hair cat. The animals of both healthy group and asthmatic group were fasted approximately $8 \mathrm{hr}$ for general anesthesia. The propofol (Provive ${ }^{\circledR}$ 1\%, Myungmoon Pharm. Co., Ltd., Seoul, Korea) was injected rapidly (6 mg/ kg, IV) for induction. The tracheal tube was intubated, and anesthesia was maintained with isoflurane (Ifran ${ }^{\circledR}$, Hana Pharm. Co., Ltd., Seoul, Korea) and oxygen. Heart rate, respiratory rate, body temperature and end tidal carbon dioxide (EtCo2) were monitored continuously during anesthesia. Studies were performed with 4 channels CT scanner (Lightspeed Plus ${ }^{\circledR}$, General Electric Medical System, San Francisco, CA, U.S.A.). The CT images of study were obtained in sternal recumbency. CT power injector (Liebel-Flarsheim ${ }^{\circledR}$, Mallinckrodt, Cincinnati, OH, U.S.A.) was used for control injection of iohexol (Omnihexol ${ }^{\circledR}$, Korea United Pharm. Inc., Seoul, Korea) as contrast media. Infused contrast media were $600 \mathrm{mg} \mathrm{I} / \mathrm{kg}$. Single breathe hold technique was performed that airway pressure was maintained at $15 \mathrm{~cm}$ of water to the airway during the CT scan. Computed tomography was performed using HRCT: $1.25 \mathrm{~mm}$ thick transverse images spaced $5 \mathrm{~mm}$ apart. CT images were acquired and reconstructed using a bone algorithm. The gantry rotation time was $1 \mathrm{sec}$, tube potential was $\mathrm{kV} 120$, and tube current was mAs 200 .

Analysis of DICOM CT images was performed using a commercially viewing and analysis software (Spectra ${ }^{\circledR}$, Infinitt Healthcare Co., Ltd., Seoul, Korea). All measurements were obtained on contrast enhanced transverse images, using moderately edge enhancing reconstruction algorithm at the lung window (window width $=1,500$ Hounsfield unit $(\mathrm{HU})$, window level $=-600$ $\mathrm{HU})$. All measurements were performed with image magnification of $1,000 \%$.

Bronchial lumen, entire bronchial diameter and adjacent arterial diameter were measured in four locations: the cranial part of left cranial, left caudal, right cranial and right caudal lung lobes. The right middle lung lobe was excluded, because the bronchus of the right middle lung lobe was visible longitudinally in transverse image. The caudal part of the left cranial lobe was also excluded due to lack of reproducibility and image quality. A second segmental bronchus from the primary bronchus to each lobe was evaluated. The second bronchus that could be best seen was measured. Pulmonary artery, bronchial lumen and whole bronchial diameter were measured for the entire hyper-dense region associated with wall and artery (Fig. 1). All measurements were tried to caliper bronchi or arteries that were mostly circular. However, if circular bronchus and artery were not identified, oval bronchus and artery were measured for smallest diameters to avoid any effect of obliquity in determining the exact diameter. By measuring the overall bronchial diameter, bronchial lumen and adjacent artery, a total of three values (BA ratio, TD ratio and TA ratio) were calculated (Fig. 2). Because the bronchial wall thickness had relatively small values than other values, directly measuring of bronchial wall thickness could be erroneous. Therefore, wall thickness (T) was derived indirectly from overall (D) and luminal (L) diameter of bronchus $(\mathrm{T}=(\mathrm{D}-\mathrm{L}) / 2)$.

All calculations in statistical analyses were performed using non-commercial software ( $\mathrm{R}$, The R Foundation) and commercial software (SPSS 19, SPSS Inc., Chicago, IL, U.S.A.). One way analysis of variation (ANOVA) was performed to identify differences between each lobe (cranial part of left cranial, left caudal, right cranial and right caudal) in healthy group. A Welch two sample $t$-test was used to compare difference between male and female. Linear regression analysis was used to identify correlations with age and ratios. A Welch two sample $t$-test was used to compare difference between healthy group and clinically asthmatic group. Receiver operating characteristic curve was created, and area under curve was calculated. Sensitivity, specificity, positive predictive value and negative predictive value were calculated using the standard approaches at various cut-off ratios. All results were reported as mean \pm standard deviation. For all statistical analyses, a $P$ value of less than 0.05 was considered significant. 


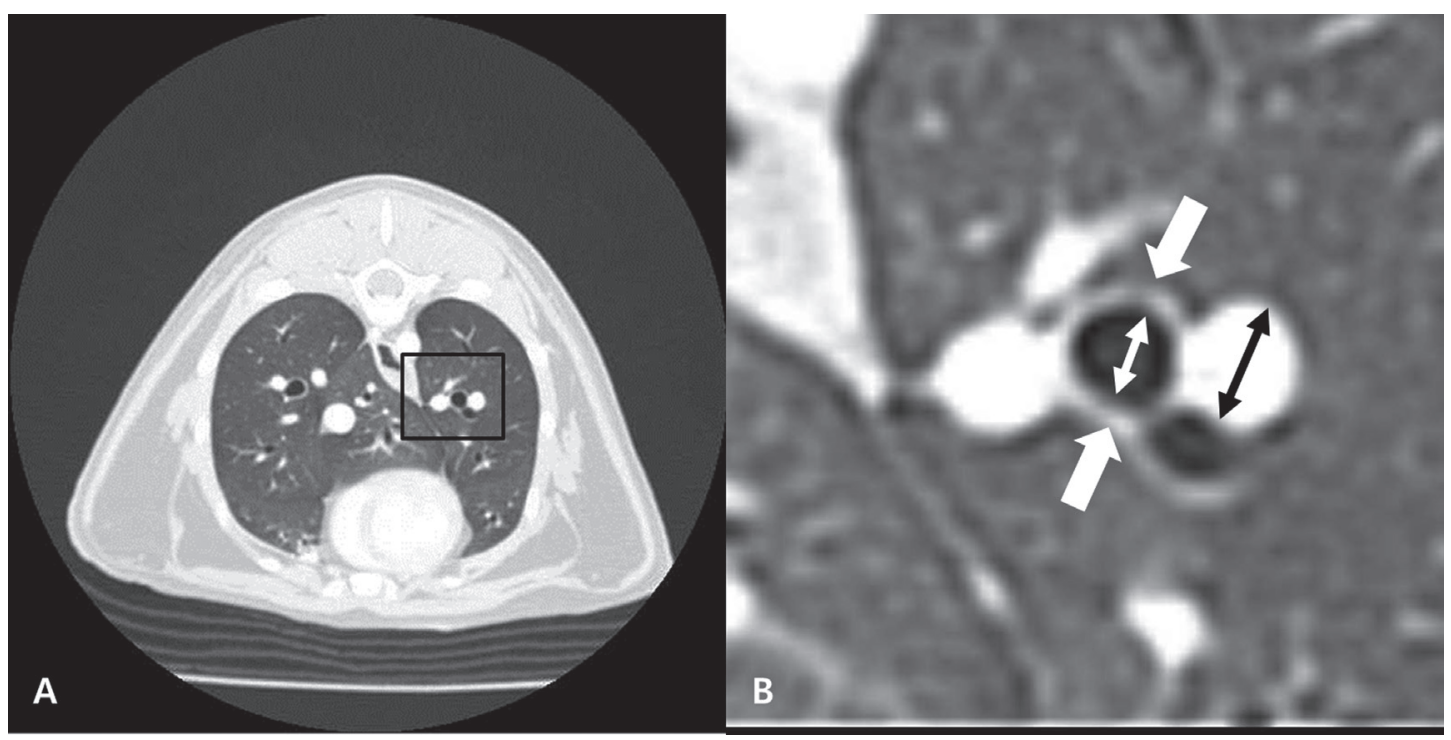

Fig. 1. High resolution computed tomography image of the caudal lung lobe. Whole transverse image (A) and magnified image (B). The whole diameter (thick white arrows) and internal diameter (thin white arrow) of caudal bronchus and the diameter of the adjacent pulmonary artery (black arrow) in transverse image were measured. The patient's left is to the right of the image.

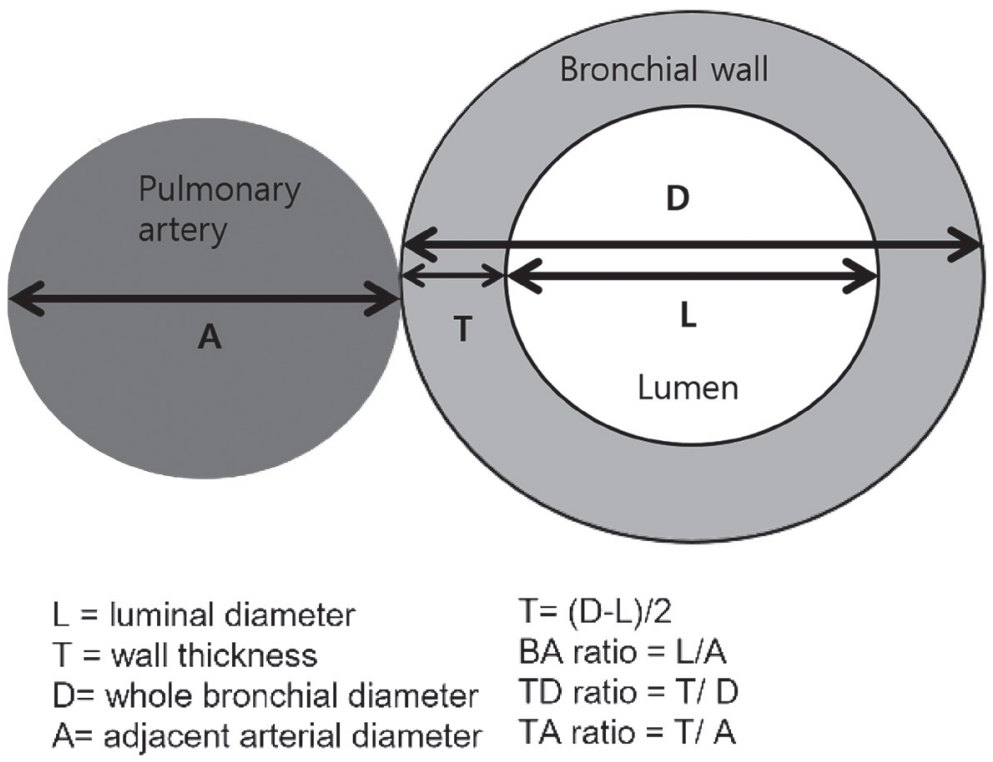

Fig. 2. Measurement schemes of bronchial lumen, wall thickness and adjacent pulmonary artery. The calculation of bronchial wall thickness was derived indirectly from overall and luminal diameter of bronchus $(\mathrm{T}=(\mathrm{D}-\mathrm{L}) / 2)$.

\section{RESULTS}

Sixty four bronchi and arteries were obtained from heathy 16 cats, and 16 bronchi and arteries were obtained from 4 asthmatic cats. In obtained image, BA ratio, TD ratio and TA ratio of healthy group and asthmatic group were calculated. The calculated indices from healthy cats and asthmatic cats are described at Table 1. TD ratio and TA ratio in asthmatic cats were significantly greater than those in healthy cats $(P<0.05)$. But, BA ratio showed no significant difference between asthmatic cats and healthy cats $(P=0.24)$. There were no significant differences in TD ratio and TA ratio between each lobe. And, there were no significant differences in TD ratio and TA ratio in either ages or gender.

Given these findings, a receiver operating characteristic curve (ROC) of TD ratio and TA ratio was created using the data from healthy group and asthmatic group (Fig. 3). The area under curve (AUC) of TD ratio was 0.869, and AUC of TA ratio was 0.945. The both AUCs of TD ratio and TA ratio were significantly greater than $0.5(P<0.05)$. Cut-off ratio of 0.203 was best balanced with 
Table 1. Mean of each index measured in clinically healthy cats and clinically asthmatic cats

\begin{tabular}{lccc}
\hline & $\begin{array}{c}\text { Healthy group } \\
(\mathrm{n}=16)\end{array}$ & $\begin{array}{c}\text { Asthmatic group } \\
(\mathrm{n}=4)\end{array}$ & $P$-value \\
\hline BA ratio & $0.86 \pm 0.12$ & $0.93 \pm 0.21$ & $P=0.24$ \\
TD ratio & $0.18 \pm 0.02$ & $0.22 \pm 0.02$ & $P<0.05$ \\
TA ratio & $0.25 \pm 0.05$ & $0.37 \pm 0.06$ & $P<0.05$ \\
\hline
\end{tabular}

BA ratio, bronchial lumen to artery ratio; TD ratio, bronchial wall to whole bronchial diameter ratio; TA ratio, bronchial wall to artery ratio.

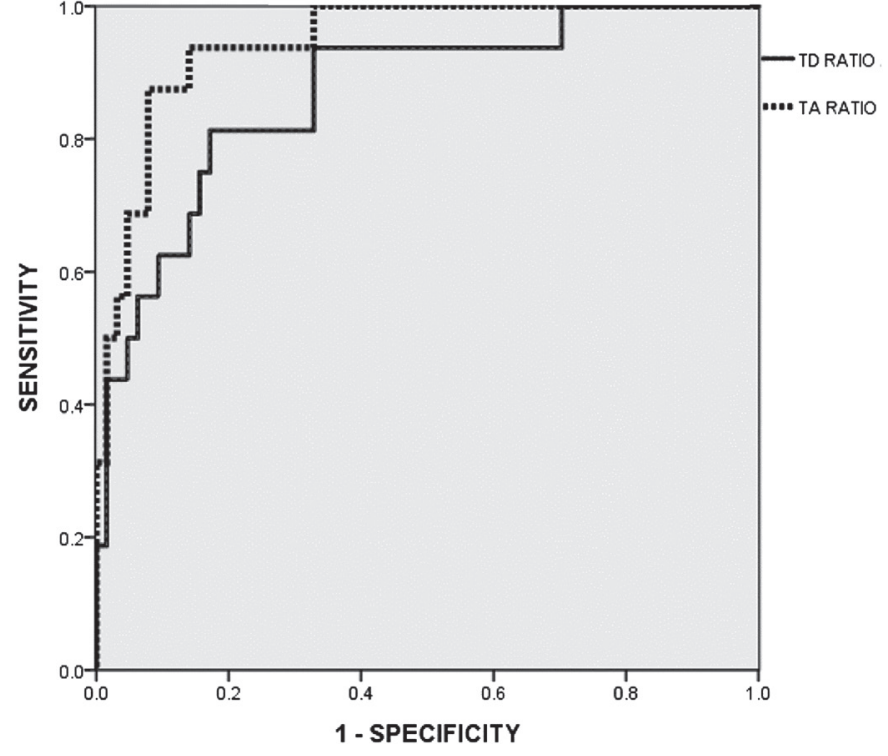

Fig. 3. Receiver operating characteristic curve of the TD ratio and TA ratio in cats with asthma and healthy cats. Solid line represents TD ratio, and dotted line represents TA ratio. The both TD ratio and TA ratio were significantly different from $0.5(P<0.05)$.

Table 2. Result using various cut-off ratios to predict bronchial wall thickening in multiple indices

\begin{tabular}{cccccccccc}
\hline Cut-off ratio & TP & TN & FP & FN & Sens & Spec & PPV & NPV & Accuracy \\
\hline TD ratio & & & & & & & & & \\
0.194 & 14 & 43 & 21 & 2 & 0.875 & 0.6729 & 0.4000 & 0.956 & 0.7125 \\
0.203 & 13 & 52 & 12 & 3 & 0.812 & 0.8125 & 0.5200 & 0.945 & 0.8125 \\
0.205 & 12 & 53 & 11 & 4 & 0.750 & 0.8281 & 0.5217 & 0.930 & 0.8125 \\
\hline TA ratio & & & & & & & & & \\
0.303 & 15 & 55 & 9 & 1 & 0.938 & 0.859 & 0.6250 & 0.9821 & 0.875 \\
0.316 & 14 & 59 & 5 & 2 & 0.875 & 0.922 & 0.7364 & 0.9672 & 0.9125 \\
0.331 & 11 & 60 & 4 & 5 & 0.688 & 0.938 & 0.7333 & 0.9230 & 0.8875 \\
\hline
\end{tabular}

TD ratio, bronchial wall to whole bronchial diameter ratio; TA ratio, bronchial wall to artery ratio TP, true positive; TN, true negative; FP, false positive; FN, false negative; Sens, sensitivity; Spec, specificity; PPV, positive predictive value; NPV, negative predictive value.

sensitivity $81.3 \%$ and specificity $81.3 \%$ in TD ratio. Cut-off ratio of 0.316 was best balanced with sensitivity $87.5 \%$ and specificity $92.2 \%$ in TA ratio (Table 2 ).

\section{DISCUSSION}

As a result, the TD ratio and TA ratio in cats with asthma were significantly greater than those in clinically healthy cats. However, the BA ratio was not significantly different between the healthy group and the asthmatic group. That is because, if the whole diameters of bronchi in feline asthma are the same, the luminal diameters will be smaller due to bronchial wall thickening. If the BA ratio is measured in lesions, such as bronchiectasis, it is considered that BA ratio may be different between asthmatic cats from healthy cats.

The AUC of TD ratio had relatively high accuracy in predicting bronchial wall thickening in asthma patients, with a value of 0.869 . The AUC of TA ratio was 0.945 , which was greater than that of TD ratio. Therefore, TA ratio is a more accurate index to detect bronchial wall thickening in asthmatic patients than TD ratio. The TD ratio cut off value of 0.203 had the best balance of sensitivity and specificity, while the TA ratio cut off value of 0.316 had the best. Therefore, to determine the bronchial wall thickening in cats with asthma, TA ratio and cut-off threshold of TD ratio could be used.

Several studies have evaluated the bronchial wall thickness in human medicine. Since bronchial wall thickness can be changed according to airway generation, we calculated the ratios of bronchial and arterial dimensions rather than their absolute values. One of the ratios was the TD ratio which was dividing bronchial wall thickness by the whole diameter of bronchus. The percentage of wall area (WA\%) has also been used in the quantification of bronchial wall thickness in human asthma patients [11]. In the case of bronchiectasis, simple measurement of bronchial wall thickness divided by bronchial luminal diameter could cause spurious 
underestimation of intrinsic bronchial wall thickening. Thus, to obtain an estimation of bronchial wall thickness, we used TA ratio, which was the ratio of mean bronchial wall thickness divided by mean diameter of the accompanying pulmonary artery used previously [5]. As a result, we found that the TA ratio was more accurate than TD ratio.

In human medicine, many studies have reported of correlation between the severity of asthma and images of bronchial changes in HRCT. The forced expiratory volume in one second (FEV1) has been reported to be correlated with WA\%. TD ratio and WA\% have been reported to be negatively correlated with carbon monoxide transfer coefficient [9]. In veterinary medicine, bronchial wall thickness ratios will be helpful to assess the severity of feline asthma, because it is quantitative. However, indexing for the severity of asthma, such as FEV1, in human medicine has not been established in veterinary medicine.

The limitation of this study is number of asthmatic cats. Furthermore, the breed of cats used in this study was not diverse. Therefore, it was difficult to compare difference between breeds. Research studies are needed to determine the differences in normal ranges of bronchial wall for a variety of feline breeds. For example, in brachycephalic breed cat, such as Persian cat, bronchial wall can be thicker than other breeds, even though there is no clinical sign. Dogs with brachycephalic airway syndrome may contribute to the loss of small airway wall rigidity [6]. Based on this finding, the BA ratio in brachycephalic breed dogs is found to be different from that in non-brachycephalic dogs [20]. In Persian cat, brachycephalic syndrome including stenotic nares and elongated soft palate has been reported [10]. Therefore, research studies on feline brachycephalic breeds are needed in the future.

For the first time, this study reported the evaluation of bronchial wall thickness of normal cats and clinically asthmatic cats using HRCT. Our results suggest that quantification indices of bronchial wall thickness, such as TD ratio and TA ratio, can be used to diagnose feline asthma syndrome and other diseases involving bronchial wall thickening. In conclusion, HRCT is a useful modality to quantify bronchial wall thickness to diagnose diseases involving the thickening of bronchial wall, including feline asthma.

\section{REFERENCES}

1. Adamama-Moraitou, K. K., Patsikas, M. N. and Koutinas, A. F. 2004. Feline lower airway disease: a retrospective study of 22 naturally occurring cases from Greece. J. Feline Med. Surg. 6: 227-233. [Medline] [CrossRef]

2. Brown, P. J., Greville, H. W. and Finucane, K. E. 1984. Asthma and irreversible airflow obstruction. Thorax 39: 131-136. [Medline] [CrossRef]

3. Burrows, B., Martinez, F. D., Halonen, M., Barbee, R. A. and Cline, M. G. 1989. Association of asthma with serum IgE levels and skin-test reactivity to allergens. N. Engl. J. Med. 320: 271-277. [Medline] [CrossRef]

4. Corcoran, B. M., Foster, D. J. and Fuentes, V. L. 1995. Feline asthma syndrome: a retrospective study of the clinical presentation in 29 cats. J. Small Anim. Pract. 36: 481-488. [Medline] [CrossRef]

5. de Jong, P. A., Ottink, M. D., Robben, S. G., Lequin, M. H., Hop, W. C., Hendriks, J. J., Paré, P. D. and Tiddens, H. A. 2004. Pulmonary disease assessment in cystic fibrosis: comparison of CT scoring systems and value of bronchial and arterial dimension measurements. Radiology 231: 434-439. [Medline] [CrossRef]

6. De Lorenzi, D., Bertoncello, D. and Drigo, M. 2009. Bronchial abnormalities found in a consecutive series of 40 brachycephalic dogs. J. Am. Vet. Med. Assoc. 235: 835-840. [Medline] [CrossRef]

7. Dunnill, M. S. 1960. The pathology of asthma, with special reference to changes in the bronchial mucosa. J. Clin. Pathol. 13: 27-33. [Medline] [CrossRef]

8. Hargreave, F. E., Ryan, G., Thomson, N. C., O’Byrne, P. M., Latimer, K., Juniper, E. F. and Dolovich, J. 1981. Bronchial responsiveness to histamine or methacholine in asthma: measurement and clinical significance. J. Allergy Clin. Immunol. 68: 347-355. [Medline] [CrossRef]

9. Little, S. A., Sproule, M. W., Cowan, M. D., Macleod, K. J., Robertson, M., Love, J. G., Chalmers, G. W., McSharry, C. P. and Thomson, N. C. 2002. High resolution computed tomographic assessment of airway wall thickness in chronic asthma: reproducibility and relationship with lung function and severity. Thorax 57: 247-253. [Medline] [CrossRef]

10. Malik, R., Sparkes, A. and Bessant, C. 2009. Brachycephalia--a bastardisation of what makes cats special. J. Feline Med. Surg. 11: 889-890. [Medline] [CrossRef]

11. Niimi, A., Matsumoto, H., Amitani, R., Nakano, Y., Mishima, M., Minakuchi, M., Nishimura, K., Itoh, H. and Izumi, T. 2000. Airway wall thickness in asthma assessed by computed tomography. Relation to clinical indices. Am. J. Respir. Crit. Care Med. 162: 1518-1523. [Medline] [CrossRef]

12. Norris Reinero, C. R., Decile, K. C., Berghaus, R. D., Williams, K. J., Leutenegger, C. M., Walby, W. F., Schelegle, E. S., Hyde, D. M. and Gershwin, L. J. 2004. An experimental model of allergic asthma in cats sensitized to house dust mite or bermuda grass allergen. Int. Arch. Allergy Immunol. 135: 117-131. [Medline] [CrossRef]

13. Padrid, P. 2000. Feline asthma. Diagnosis and treatment. Vet. Clin. North Am. Small Anim. Pract. 30: 1279-1293. [Medline] [CrossRef]

14. Paganin, F., Trussard, V., Seneterre, E., Chanez, P., Giron, J., Godard, P., Sénac, J. P., Michel, F. B. and Bousquet, J. 1992. Chest radiography and high resolution computed tomography of the lungs in asthma. Am. Rev. Respir. Dis. 146: 1084-1087. [Medline] [CrossRef]

15. Peat, J. K., Woolcock, A. J. and Cullen, K. 1987. Rate of decline of lung function in subjects with asthma. Eur. J. Respir. Dis. 70: 171-179. [Medline]

16. Reid, L. E., Dillon, A. R., Hathcock, J. T., Brown, L. A., Tillson, M. and Wooldridge, A. A. 2012. High-resolution computed tomography bronchial lumen to pulmonary artery diameter ratio in anesthetized ventilated cats with normal lungs. Vet. Radiol. Ultrasound 53: 34-37. [Medline] [CrossRef]

17. Senéterre, E., Paganin, F., Bruel, J. M., Michel, F. B. and Bousquet, J. 1994. Measurement of the internal size of bronchi using high resolution computed tomography (HRCT). Eur. Respir. J. 7: 596-600. [Medline] [CrossRef]

18. Szabo, D., Sutherland-Smith, J., Barton, B., Rozanski, E. A. and Taeymans, O. 2015. Accuracy of a computed tomography bronchial wall thickness to pulmonary artery diameter ratio for assessing bronchial wall thickening in dogs. Vet. Radiol. Ultrasound 56: 264-271. [Medline] [CrossRef]

19. Webb, W. R., Gamsu, G., Wall, S. D., Cann, C. E. and Proctor, E. 1984. CT of a bronchial phantom. Factors affecting appearance and size measurements. Invest. Radiol. 19: 394-398. [Medline] [CrossRef]

20. Won, S., Lee, A., Choi, J., Choi, M. and Yoon, J. 2015. Computed tomographic bronchioarterial ratio for brachycephalic dogs without pulmonary disease. J. Vet. Sci. 16: 221-224. [Medline] [CrossRef] 